\title{
Elasticity-Based Mechanism for the Collective Motion of Self-Propelled Particles with Springlike Interactions: A Model System for Natural and Artificial Swarms
}

\author{
Eliseo Ferrante \\ Laboratory of Socioecology and Social Evolution, KU Leuven, B-3000 Leuven, Belgium
}

\author{
Ali Emre Turgut \\ University of Turkish Aeronautical Association, TR-06790 Ankara, Turkey
}

Marco Dorigo

IRIDIA, Université Libre de Bruxelles, B-1050 Brussels, Belgium and Department of Computer Science, University of Paderborn, D-33102 Paderborn, Germany

\author{
Cristián Huepe \\ CHuepe Labs, 954 West 18th Place, Chicago, Illinois 60608, USA; Max Planck Institute for the Physics of Complex Systems, D-01187 \\ Dresden, Germany; and Northwestern Institute on Complex Systems, Northwestern University, Evanston, Illinois 60208, USA
}

(Received 31 December 2012; published 26 December 2013)

\begin{abstract}
We introduce an elasticity-based mechanism that drives active particles to self-organize by cascading self-propulsion energy towards lower-energy modes. We illustrate it on a simple model of self-propelled agents linked by linear springs that reach a collectively rotating or translating state without requiring aligning interactions. We develop an active elastic sheet theory, complementary to the prevailing active fluid theories, and find analytical stability conditions for the ordered state. Given its ubiquity, this mechanism could play a relevant role in various natural and artificial swarms.
\end{abstract}

DOI: 10.1103/PhysRevLett.111.268302

PACS numbers: 82.39.Rt, 83.50.-v, 87.17.Jj, 89.75.Fb

Animal groups that move together, such as bacterial colonies, insect swarms, bird flocks, or fish schools [1-6], are all examples of biological systems displaying collective motion (CM). In recent years, the dynamics of such systems (referred to here generically as swarms) has been the subject of intense research [6-9]. A number of theoretical models have been introduced to study swarms and to develop control rules that achieve similar coordinated collective dynamics in groups of autonomous robots [8,9]. Despite this proliferation of algorithms, there is still no clear understanding of the different underlying mechanisms that can lead a group of self-propelled agents to selforganize into CM. While most models assume aligning interactions that produce heading angle consensus, numerical and experimental evidence suggests that $\mathrm{CM}$ can be achieved without these [10-12], motivating our search for alternative mechanisms. We develop here an active elastic sheet (AES) theory that reveals one of these mechanisms, which is fundamentally different from the well-studied ferromagneticlike alignment mechanism introduced in [13], and results instead from standard elasticity dynamics.

The current CM paradigm has been strongly influenced by the seminal work of Vicsek et al. [13], which introduced a minimal model for flocking, the Vicsek model, that has become a referent in the field [7-9]. This model describes a group of point particles advancing at a fixed common speed, only coupled through alignment interactions that steer them towards the mean heading direction of all particles within a given radius [13-15]. In this framework, a swarm can be viewed as a group of self-propelled spins with aligning interactions, described by a variation of the $X Y$ model [16] where spins advance in their pointing direction rather than remaining affixed to a lattice. In the continuous limit, this system becomes a fluid of selfpropelled spins that follows the hydrodynamic theory developed in [17-20]. (Note that we will not consider here self-propelled swimmers, which belong to a different class of systems where the embedding fluid's hydrodynamics must be considered [21-26].) More recently, some models that do not rely on explicit alignment interactions have also been introduced. In [10], for example, CM is driven by escape-pursuit interactions only, in [27] by inelastic collisions between isotropic agents, and in [11] and [12] by short-range radial forces coupled to each agent's turning dynamics. Given that Vicsek-like algorithms rely on explicit alignment rules to achieve CM $[5,13,28,29]$, it was initially surprising that such systems could selforganize without them. While it can be argued that all these models include at least an implicit alignment interaction, it remains unclear if they are all driven to $\mathrm{CM}$ by the same underlying heading consensus mechanism and to what extent agents must exchange orientation information, either explicitly or implicitly, to achieve CM.

In this Letter, we introduce a CM mechanism that is based on a very different paradigm: the emergence and 
growth of regions of coherent motion due to standard elasticity processes. We explore this mechanism by introducing a simple two-dimensional AES model with springlike interactions between neighboring agents and no explicit alignment, which describes what we refer to as an active solid or an active crystal.

We define the AES model as a system of $N$ agents on a two-dimensional plane, where the position $\vec{x}_{i}$ and orientation $\theta_{i}$ of agent $i$ follow the overdamped equations of motion,

$$
\begin{aligned}
& \dot{\vec{x}}_{i}=v_{0} \hat{n}_{i}+\alpha\left[\left(\vec{F}_{i}+D_{r} \hat{\xi}_{r}\right) \cdot \hat{n}_{i}\right] \hat{n}_{i}, \\
& \dot{\theta}_{i}=\beta\left[\left(\vec{F}_{i}+D_{r} \hat{\xi}_{r}\right) \cdot \hat{n}_{i}^{\perp}\right]+D_{\theta} \xi_{\theta} .
\end{aligned}
$$

Here, $v_{0}$ is the forward biasing speed that induces selfpropulsion (injecting energy at the individual particle level), $\hat{n}_{i}$ and $\hat{n}_{i}^{\perp}$ are two unit vectors pointing parallel and perpendicular to the heading direction of agent $i$, and parameters $\alpha$ and $\beta$ are the inverse translational and rotational damping coefficients, respectively. The total force over agent $i$ is given by $\vec{F}_{i}=$ $\sum_{j \in S_{i}}\left(-k / l_{i j}\right)\left(\left|\vec{r}_{i j}\right|-l_{i j}\right) \vec{r}_{i j} /\left|\vec{r}_{i j}\right|$ (with $\vec{r}_{i j}=\vec{x}_{j}-\vec{x}_{i}$ ), a sum of linear springlike forces with equilibrium distances $l_{i j}$ and spring constants $k / l_{i j}$. Each set $S_{i}$ contains all agents interacting with agent $i$ and remains fixed throughout the integration. This system is thus akin to a spring-mass model of elastic sheet [30] where masses are replaced by selfpropelled agents that turn according to $\vec{F}_{i} \cdot \hat{n}_{i}^{\perp}$ and move forward or backward following $\vec{F}_{i} \cdot \hat{n}_{i}$ and their selfpropulsion. We include actuation noise (fluctuations of the individual motion) by adding $D_{\theta} \xi_{\theta}$ to the heading angle, where $D_{\theta}$ is the noise strength coefficient and $\xi_{\theta}$ a random variable with standard, zero-centered normal probability distribution of variance 1 . We include sensing noise (errors in the measured forces) by adding $D_{r} \hat{\xi}_{r}$ to $\vec{F}_{i}$, with $D_{r}$ the noise strength coefficient and $\hat{\xi}_{r}$ a randomly oriented unit vector. We chose two different noise sources to include variants known to produce different effects in other CM systems [8,31]. The degree of alignment is monitored by the usual polarization order parameter $\psi=\left\|\sum_{i=1}^{N} \hat{n}_{i}\right\| / N$, where $\psi=1$ if all agents are perfectly aligned and $\psi=0$ if they are randomly oriented or rotating about the group's barycenter.

The AES model was designed to achieve CM under conditions that are in many ways opposite to the Vicsek ones. While Vicsek agents only sense their relative heading angles, AES agents only sense their relative positions. While the Vicsek dynamics requires changing interacting neighbors over time to achieve long-range order [14,17,32], the AES case has virtual springs connecting the same agents throughout the dynamics. Furthermore, although both models describe overdamped systems, Vicsek particles relax instantaneously to the next desired heading angle while AES agents turn following Eq. (2), which we found is necessary for achieving CM. In sum, the AES model explores a limit situation of elasticlike collective dynamics with only position-based interactions, much like the Vicsek model explores the fluidlike limit with only orientation-based interactions. Note that we observed the same qualitative dynamics when model conditions were relaxed by using finite-range attraction-repulsion forces that allow neighbor exchanges when links are broken (violating the structure's topological integrity). Our results could thus be relevant for a broad range of systems.

We integrated Eqs. (1) and (2) numerically using a standard Euler method. All simulations below were carried out with $\alpha=0.01, \beta=0.12, v_{0}=0.002$, and $d t=0.1$.

Figure 1 presents three runs of the AES model. Column A displays the dynamics of an hexagonal active crystal composed of $N=91$ agents. At $t=0$ (panel A1), randomly oriented agents are placed on a perfect hexagonal lattice, separated by $d_{A}=0.65$. Nearest neighbors are connected by springs with natural length $l=d_{A}$ and spring constant $k / l=5 / 0.65$. Only sensing noise is considered $\left(D_{r} \approx 0.158, D_{\theta}=0\right)$, but results remain qualitatively unchanged for other subcritical noise combinations. As time advances, growing regions of coherent motion develop, deforming the whole structure (A2) until the group starts translating or rotating collectively. Here, the system converges to a state that rotates while translating (A3). Note that rotating states have higher elastic energy, since inner

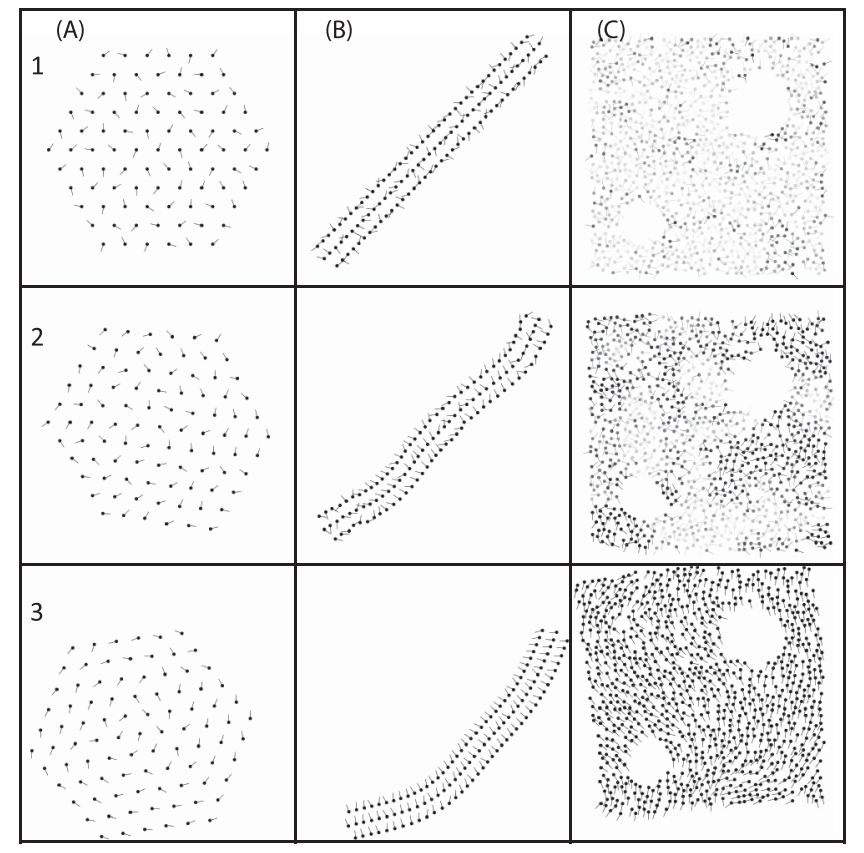

FIG. 1. Active elastic sheet simulations of Eqs. (1) and (2). (A) Hexagonal active crystal at $t=0$ (A1), 240 (A2), and 1700 (A3). (B) Rodlike active crystal at $t=0$ (B1), 400 (B2), and 1700 (B3). (C) Active solid at same times as column B; darker agents symbolize higher local alignment. See Supplemental Material for movies of these simulations [33]. 
and outer shells cannot move at the same $v_{0}$ speed and must be sped up or slowed down by elastic forces. These states are metastable and can relax to lower-energy translating solutions. We show one here to illustrate its dynamics, which cannot be attained by the Vicsek model.

Column B displays an active elastic rod, comprised of $N=118$ agents arranged into three rows, with the same noise as in A. It is generated (B1) by placing randomly oriented agents with nearest-neighbor distances $d_{B}=0.32$ (within rows) and $d_{B}^{*}=0.58$ (between rows), linking all agents separated by $d<1$ with springs of natural length $d$ and spring constants $k / l=1 / d$. Here again, growing regions of coherent deformation emerge (B2) until CM is attained and the rod starts moving (B3). Since the first bending mode has the largest final deformation, a collective heading direction perpendicular to the rod's axis is favored. This opens the possibility of controlling the self-organized $\mathrm{CM}$ direction by arranging self-propelled agents into different formations.

Column C shows $N=891$ agents forming an active solid (given the irregular agent positions) with two holes. To construct it, we distribute agents at random, homogeneously within the structure, connecting all agents separated by $d<1$, as in column B, but using $k / l=5 / d$. Here $D_{r}=D_{\theta}=0$, but equivalent dynamics are observed for small enough values. To highlight ordered regions, each agent's darkness is displayed proportional to the local order, defined as $\psi$ but summing only over the focal agent and those linked to it, instead of the whole system. Initially, most of the structure appears in light gray, since agents are randomly oriented (C1). As time advances, coherent (darker) regions grow $(\mathrm{C} 2)$ until the whole structure starts moving when agents become sufficiently aligned (C3).

The AES model displays a discontinuous order-disorder transition similar to that in the Vicsek model [29]. Figure 2 examines it as a function of noise for the hexagonal active crystal on Fig. 1(A) and for larger $(N=547, N=32137)$ hexagonal configurations with identical parameters. We performed 30 runs per noise value ( 80 near the transition), storing $2000 \psi$ values per run (every 500 time steps after the initial $10^{6}$ ). Bottom panels show the mean and local maxima of the $\psi$ distributions. As we increase noise, $\psi$ jumps from an ordered state where agents align to a disordered state with random headings. Top panels show that the distributions of $\psi$ values become more bimodal in the transition region for larger systems. These numerical results show that the AES transition is first order with a bistable region for both types of noise. Furthermore, the system supports long-range order at nonzero noise values, as evidenced by the bottom panel insets. These plot $\psi_{r}^{*} \equiv\left\langle\psi\left(D_{r}=0.51\right)\right\rangle$ and $\psi_{r}^{*} \equiv$ $\left\langle\psi\left(D_{r}=0.51\right)\right\rangle \quad$ vs $N$ in $\log -\log$ scale with up to $N \approx 10^{5}$ agents, showing that global order is preserved as $N \rightarrow \infty$.
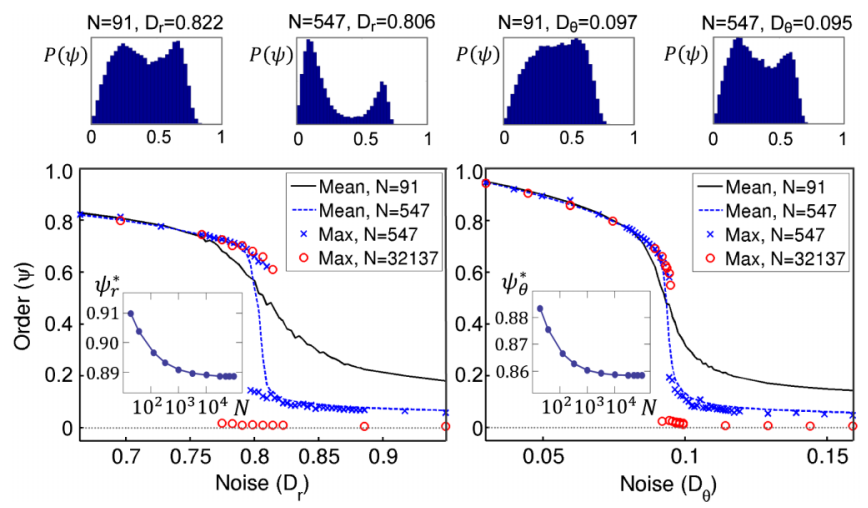

FIG. 2 (color online). Order parameter $\psi$ vs positional sensory noise $D_{r}$ and angular actuation noise $D_{\theta}$ for hexagonal active crystals with $N=91$ (same as on Fig. 1, panel A1), $N=547$, and $N=32137$ agents. Both cases display a first-order transition with bistable region. Bottom panels show the mean and local maxima of the distribution of $\psi$ values obtained in simulations. Top panels show these distributions in the transition region for two system sizes. Insets plot $\langle\psi\rangle$ vs system size at fixed noise level.

A relevant feature of the AES model is that we can use a continuous elastic sheet approximation to perform analytical calculations. We follow this approach to carry out a standard linear stability analysis [30] of the translating $\mathrm{CM}$ state in the zero noise case. We begin by writing the elastic forces $\vec{F}=\left(F_{x}, F_{y}\right)$ that result from small displacements $\vec{u}=\left(u_{x}, u_{y}\right)$ of points on the membrane with respect to their equilibrium positions

$$
\begin{aligned}
& F_{x}=(\lambda+2 \mu) \frac{\partial^{2} u_{x}}{\partial x^{2}}+\mu \frac{\partial^{2} u_{x}}{\partial y^{2}}+(\lambda+\mu) \frac{\partial^{2} u_{y}}{\partial x \partial y}, \\
& F_{y}=(\lambda+2 \mu) \frac{\partial^{2} u_{y}}{\partial y^{2}}+\mu \frac{\partial^{2} u_{y}}{\partial x^{2}}+(\lambda+\mu) \frac{\partial^{2} u_{x}}{\partial x \partial y},
\end{aligned}
$$

where the elastic constants are the Lamé parameter $\lambda$ and shear modulus $\mu$ [30]. We then linearize Eqs. (1) and (2) around an equilibrium solution with undeformed membrane and all agents moving at speed $v_{0}$ in the $\hat{x}$ direction, obtaining $\dot{u}_{x}=\alpha F_{x}, \dot{u}_{y}=v_{0} \varphi$, and $\dot{\varphi}=\beta F_{y}$, where $\varphi$ denotes perturbations to the $\theta=0$ equilibrium heading angle. Casting these expressions in Fourier space with wave-vector components $\left(k_{x}, k_{y}\right)$, we can write the perturbation dynamics in matrix form and compute its eigenvalues $\Lambda$ to determine stability. These are found to satisfy the characteristic equation $\Lambda^{3}+C_{2} \Lambda^{2}+C_{1} \Lambda+C_{0}=0$, with

$$
\begin{gathered}
C_{0}=\alpha \beta \mu v_{0}(\lambda+2 \mu)\left[k_{x}^{2}+k_{y}^{2}\right]^{2}, \\
C_{1}=\beta v_{0}\left[\mu k_{x}^{2}+(\lambda+2 \mu) k_{y}^{2}\right], \\
C_{2}=\alpha\left[(\lambda+2 \mu) k_{x}^{2}+\mu k_{y}^{2}\right] .
\end{gathered}
$$


Using Routh's stability criterion (here given by $C_{1} C_{2}>C_{0}$ ) [34], we find that the system is stable if $\alpha \beta v_{0}(\lambda+\mu)^{2} k_{x}^{2} k_{y}^{2}>0$, which is always verified. We conclude that translating CM solutions are always linearly stable. This is not the case, however, for most variations of the AES model. For example, if we consider a constant speed algorithm by setting $\alpha=0$, the characteristic polynomial becomes $\Lambda^{3}+\beta v_{0}\left[\mu k_{x}^{2}+(\lambda+2 \mu) k_{y}^{2}\right] \Lambda=0$, which only has null or imaginary solutions. Linear perturbations will therefore not dampen out, but produce instead permanent oscillations. Numerical simulations confirm that, even for zero noise and starting from a perfectly aligned initial condition, the group loses order as agents rotate in place instead of aligning.

We now characterize the nonlinear energy cascading mechanism that drives the AES model to self-organize. Figure 3 presents the energy dynamics of an hexagonal active crystal simulation with $N=91$ and zero noise that converges to a translating solution. Top panels display the total kinetic and potential energies as a function of time. Panel C shows the spectral decomposition of the latter into its elastic modes, listed in order of growing energy and without accounting for degeneracies. It is produced by first computing all 182 elastic normal modes numerically (without considering agent orientations or self-propulsion) and then expanding the dynamics into this basis. The initial condition is set as in Fig. 1(A), with zero potential energy and kinetic energy $E_{k}=N v_{0}^{2} / 2=1.82 \times 10^{-4}$ (setting the

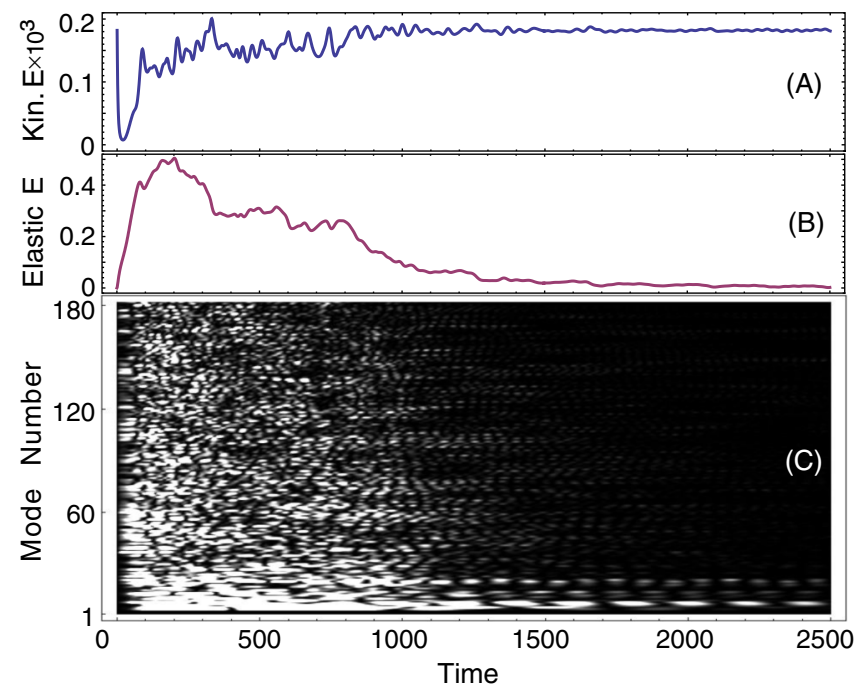

FIG. 3 (color online). Kinetic energy (A), elastic energy (B), and spectral decomposition of the elastic energy $(\mathrm{C})$ as a function of time for an hexagonal $N=91$ active crystal simulation (with zero noise and same initial condition as in panel A1 of Fig. 1) that converges to the aligned state. Brighter points on $\mathrm{C}$ indicate higher energies. After an initial transient, $\mathrm{A}$ and $\mathrm{B}$ converge to their stationary values for collective translational motion. All modes display energy levels that oscillate as they decay, with higher modes decaying faster. Elastic energy flows to lower modes, producing coherent motion that eventually reaches the lowest (translational or rotational) modes. agent mass to 1). As the membrane deforms during the initial transient, potential energy grows and becomes broadly distributed over all modes (as expected for disordered systems), while kinetic energy drops. As time advances, the system rearranges itself into configurations with lower elastic energy and higher kinetic energy, eventually reaching again (now in the ordered, translating state) values close to zero and $E_{k}$, respectively. The energy of each mode oscillates while decaying, as in an underdamped oscillator, with higher modes decaying faster than lower ones. This results from a combination of standard elasticity, selfpropulsion, and the coupling between elastic forces and turning rate imposed by the AES model. Indeed, in standard damped elastic systems, higher energy modes also decay faster. Here, however, each agent is continuously injecting energy through its self-propulsion term, so motion cannot be fully dampened. Instead, modes decay by steering agents away from them, since advancing in directions that excite higher elastic modes is disfavored. Self-propulsion thus feeds energy to lower and lower modes, eventually reaching the translational or rotational mode and achieving CM. Note that, despite this mechanism, similar models may not converge to $\mathrm{CM}$ if, for example, agents inject too much energy into high-energy modes while turning (as in the aforementioned $\alpha=0$ case) or overshoot the angles that dampen these modes by instantaneously switching heading (as in $[28,29])$ instead of integrating Eq. (2).

We have identified in this Letter an alternative, elasticitybased mechanism that, in contrast to the Vicsek case, requires no exchange of heading information to achieve CM. It also requires no fluidlike mixing (i.e., switching of interacting neighbors over time) to overcome the Mermin-Wagner theorem, since it achieves long-range order at nonzero noise levels $[14,17,32,35]$. This is because the forces leading here to alignment are fundamentally different from ferromagneticlike aligning interactions, resulting instead from elastic deformations that accumulate between misaligned agents over time. We found only one other model, introduced in [11] to study the collective migration of tissue cells, that displays $\mathrm{CM}$ under similar conditions. In a version of this algorithm (designed to study active jamming) where agents only have repulsive interactions and are confined to a circular box, elastic modes were shown to be responsible for the dynamics of the jammed phase [12]. That model, however, describes a different situation (where interaction forces can displace agents sideways) and its self-organizing properties have not been fully analyzed. Further investigation of its energy dynamics should unveil if it develops CM through a similar energy-cascading mechanism. An equivalent analysis should be carried out in models that include fluid and crystalline phases $[28,36]$ to determine the role of this mechanism in their self-organizing dynamics.

Our work provides a simple approach for creating artificial swarms and suggests how to determine experimentally if elasticity- or alignment-based interactions are behind CM 
in specific systems (such as bacteria [37] or tissue cells $[11,38]$ ), without having to probe individual interactions, by analyzing if their dynamics organize into elastic modes or if perturbations propagate as in active elastic systems. A similar approach could be followed even for macroscopic animals, where experiments show that aligning interactions suffice to explain $\mathrm{CM}$ in birds [39,40], but are negligible between two fish [41] (although both results may depend on the species and regimes considered). More in general, given that some kind of attraction-repulsion interactions must be present for any group to remain cohesive and avoid overlapping, the elasticity-based mechanism could play a relevant role in a variety of swarms. It is likely that many natural systems combine alignment- and elasticity-based dynamics, effectively behaving as active aligning viscoelastic systems.

The work of C. H. was supported by the National Science Foundation under Grant No. PHY-0848755. The work of E. F. and A.E. T. was partially supported by the Fund for Scientific Research (FWO)-Flanders through the European Science Foundation (ESF) H2SWARM project. The work of E. F., A. E. T., and M. D. was partially supported by the European Union's ERC Advanced Grant (Contract No. 246939). We thank H. Chaté for useful discussions and acknowledge support by the Max Planck Institute for the Physics of Complex Systems in Dresden, Germany, through the Advanced Study Group "Statistical Physics of Collective Motion," where part of this work was conducted.

*Presently at the Laboratory of Socioecology and Social Evoution, KU Leuven, B-3000 Leuven, Belgium. † cristian@ northwestern.edu

[1] C. M. Breder, Jr., Ecology 35, 361 (1954).

[2] A. Okubo, Adv. Biophys. 22, 1 (1986).

[3] D. Grünbaum and A. Okubo, Lect. Notes Biomath 100, 296 (1994).

[4] Bacteria as Multicellular Organisms, edited by J. A. Shapiro and M. Dworkin (Oxford University, New York, 1997).

[5] I. D. Couzin, J. Krause, R. James, G. D. Ruxton, and N. R. Franks, J. Theor. Biol. 218, 1 (2002).

[6] D. J. T. Sumpter, Collective Animal Behavior (Princeton University, Princeton, NJ, 2010).

[7] A. Deutsch, G. Theraulaz, and T. Vicsek, Interface Focus 2, 689 (2012).

[8] T. Vicsek and A. Zafeiris, Phys. Rep. 517, 71 (2012).

[9] M. Brambilla, E. Ferrante, M. Birattari, and M. Dorigo, Swarm Intell. 7, 1 (2013).

[10] P. Romanczuk, I. D. Couzin, and L. Schimansky-Geier, Phys. Rev. Lett. 102, 010602 (2009).

[11] B. Szabó, G. J. Szöllösi, B. Gönci, Z. Jurányi, D. Selmeczi, and T. Vicsek, Phys. Rev. E 74, 061908 (2006).

[12] S. Henkes, Y. Fily, and M. C. Marchetti, Phys. Rev. E 84, 040301(R) (2011).

[13] T. Vicsek, A. Czirók, E. Ben-Jacob, I. Cohen, and O. Shochet, Phys. Rev. Lett. 75, 1226 (1995).
[14] A. Czirók, H. E. Stanley, and T. Vicsek, J. Phys. A 30, 1375 (1997).

[15] H. Chaté, F. Ginelli, G. Grégoire, and F. Raynaud, Phys. Rev. E 77, 046113 (2008).

[16] J. J. Binney, N. J. Dowrick, A. J. Fisher, and M. E. J. Newman, The Theory of Critical Phenomena: An Introduction to Renormalization Group (Oxford University, New York, 1992).

[17] J. Toner and Y. Tu, Phys. Rev. Lett. 75, 4326 (1995).

[18] J. Toner and Y. Tu, Phys. Rev. E 58, 4828 (1998).

[19] E. Bertin, M. Droz, and G. Grégoire, Phys. Rev. E 74, 022101 (2006).

[20] A. Peshkov, S. Ngo, E. Bertin, H. Chaté, and F. Ginelli, Phys. Rev. Lett. 109, 098101 (2012).

[21] R. A. Simha and S. Ramaswamy, Phys. Rev. Lett. 89, 058101 (2002).

[22] Y. Hatwalne, S. Ramaswamy, M. Rao, and R. A. Simha, Phys. Rev. Lett. 92, 118101 (2004).

[23] T. B. Liverpool and M. C. Marchetti, Europhys. Lett. 69, 846 (2005).

[24] D. L. Koch and G. Subramanian, Annu. Rev. Fluid Mech. 43, 637 (2011).

[25] N. Desreumaux, N. Florent, E. Lauga, and D. Bartolo, Eur. Phys. J. E 35, 1 (2012).

[26] G. Jayaraman, S. Ramachandran, S. Ghose, A. Laskar, M. S. Bhamla, P. B. Sunil Kumar, and R. Adhikari, Phys. Rev. Lett. 109, 158302 (2012).

[27] D. Grossman, I. S. Aranson, and E. B. Jacob, New J. Phys. 10, 023036 (2008).

[28] G. Grégoire, H. Chaté, and Y. Tu, Physica (Amsterdam) 181D, 157 (2003).

[29] G. Grégoire and H. Chaté, Phys. Rev. Lett. 92, 025702 (2004).

[30] A. Fetter and J. Walecka, Theoretical Mechanics of Particles and Continua, Dover Books on Physics (Dover, New York, 2003).

[31] S. T. Smith and R. M. Seugling, Precis. Eng. 30, 245 (2006).

[32] M. Aldana and C. Huepe, J. Stat. Phys. 112, 135 (2003).

[33] See Supplemental Material at http://link.aps.org/ supplemental/10.1103/PhysRevLett.111.268302 for movies of these active elastic sheet simulations.

[34] K. Ogata, Modern Control Engineering (Prentice-Hall, New York, 2001), 4th ed.

[35] N. D. Mermin and H. Wagner, Phys. Rev. Lett. 17, 1133 (1966).

[36] A. M. Menzel and H. Löwen, Phys. Rev. Lett. 110, 055702 (2013).

[37] C. Ingham and E. Jacob, BMC Microbiology 8, 36 (2008).

[38] X. Trepat, M. R. Wasserman, T. E. Angelini, E. Millet, D. A. Weitz, J. P. Butler, and J. J. Fredberg, Nat. Phys. 5, 426 (2009).

[39] A. Cavagna, I. Giardina, A. Orlandi, G. Parisi, A. Procaccini, M. Viale, and V. Zdravkovic, Animal Behaviour 76, 217 (2008).

[40] W. Bialek, A. Cavagna, I. Giardina, T. Mora, E. Silvestri, M. Viale, and A. M. Walczak, Proc. Natl. Acad. Sci. U.S.A. 109, 4786 (2012).

[41] Y. Katz, K. Tunstrom, C. C. Ioannou, C. Huepe, and I. D. Couzin, Proc. Natl. Acad. Sci. U.S.A. 108, 18720 (2011). 